Revue d'histoire de l'Amérique française

REVUE D.HISTOIRE DE L'AMÉRIQUE FRANÇAISE

\title{
Seigneur, censitaires et paysage rural : le papier-terrier de la seigneurie de la Rivière-Ouelle de 1771
}

\section{Alain Laberge}

Volume 44, numéro 4, printemps 1991

URI : https://id.erudit.org/iderudit/304925ar

DOI : https://doi.org/10.7202/304925ar

Aller au sommaire du numéro

Éditeur(s)

Institut d'histoire de l'Amérique française

ISSN

0035-2357 (imprimé)

1492-1383 (numérique)

Découvrir la revue

Citer cet article

Laberge, A. (1991). Seigneur, censitaires et paysage rural : le papier-terrier de la seigneurie de la Rivière-Ouelle de 1771. Revue d'histoire de l'Amérique française, 44(4), 567-587. https://doi.org/10.7202/304925ar
Résumé de l'article

Ce texte veut esquisser une présentation générale de la pratique des papiers-terriers au Canada et établir les apports et les limites de ces documents seigneuriaux pour l'histoire rurale de la vallée laurentienne pré-industrielle en menant une véritable analyse des données contenues dans ce type de source. En somme, il s'agit d'une contribution à une meilleure compréhension d'une pratique seigneuriale, de son support documentaire et de son potentiel pour la recherche. 


\title{
SEIGNEUR, CENSITAIRES ET PAYSAGE RURAL: LE PAPIER-TERRIER DE LA SEIGNEURIE DE LA RIVIÈRE-OUELLE DE 1771
}

\author{
ALAIN LABERGE \\ Département d' histoire \\ Université Laval
}

\section{RÉSUMÉ}

Ce texte veut esquisser une présentation générale de la pratique des papiers-terriers au Canada et établir les apports et les limites de ces documents seigneuriaux pour l'histoire rurale de la vallée laurentienne pré-industrielle en menant une véritable analyse des données contenues dans ce type de source. En somme, il s'agit d'une contribution à une meilleure compréhension d'une pratique seigneuriale, de son support documentaire et de son potentiel pour la recherche.

\section{ABSTRACT}

This paper attempts to sketch a general presentation of the practice of the seigneurial estate rolls in Canada, and to verify the importance of these documents for the rural history of the pre-industrial laurentian valley, through a thorough analysis of its content. Altogether, this study contributes to a better understanding of a seigneurial practice, of the documents it produced, and of their potential for research.

Le renouvellement récent des interprétations du monde rural de la vallée du Saint-Laurent à l'époque pré-industrielle est largement tributaire des analyses menées à petite échelle, que ce soit au niveau de la paroisse ou de la seigneurie. Du point de vue documentaire, cette perspective monographique a également le mérite d'avoir systématisé le recours à des sources connues comme les registres d'état civil et les greffes des notaires et mis en valeur bon nombre de documents plus obscurs émanant des archives paroissiales ou seigneuriales. Ainsi, les

1 Ce texte est le résultat d'une recherche post-doctorale menée grâce à une bourse du Conseil de recherches en sciences humaines du Canada. Une première version en a été présentée au congrès annuel de la Société historique du Canada à Québec en juin 1989. Je tiens à remercier Jacques Mathieu, Serge Courville, Sylvie Dépatie, Renald Lessard, Lina Gouger, Geneviève Postolec ainsi que les évaluateurs de la Revue d'histoire de l'Amérique française pour leurs judicieuses remarques. 
chercheurs n'ont pas manqué de se servir des papiers-terriers seigneuriaux, qui renferment des informations sur les tenanciers, sur leurs propriétés à l'intérieur du fief et sur les redevances envers le seigneur, afin d'éclairer certains aspects d'une administration seigneuriale ou d'examiner la répartition de la propriété foncière dans une localité donnée ${ }^{2}$.

Or, contrairement à la France où les papiers-terriers occupent une place importante dans l'étude de l'évolution du régime seigneurial en particulier comme partie essentielle de la «réaction seigneuriale» du XVIII ${ }^{e}$ siècle $^{3}-$, ce type de document demeure une source mal connue dans le contexte laurentien. En fait, il n'existe qu'une seule étude assez systématique des papiers-terriers canadiens, celle de Richard Chabot qui, déplorant la méconnaissance de ces documents, signale diverses pistes de recherche possibles à partir de deux terriers de la seigneurie de Nicolet au début du XIX ${ }^{\mathrm{e}}$ siècle ${ }^{4}$. La démarche de Chabot est très instructive et stimulante, mais l'auteur reste toutefois trop indifférent aux papiers-terriers seigneuriaux du XVIII ${ }^{\mathrm{e}}$ siècle et aux éventuelles variations de contenu d'un document à l'autre.

Cette étude vise deux objectifs principaux. D'une part, elle esquisse une présentation générale de la pratique des papiers-terriers seigneuriaux au Canada, surtout en ce qui concerne son implantation graduelle au XVIII ${ }^{e}$ siècle. D'autre part, elle cherche à établir les apports et les limites de ces papiers-terriers pour l'histoire rurale de la vallée laurentienne pré-industrielle en menant une véritable analyse des données contenues dans ce type de document. En somme, il s'agit d'une contribution à une meilleure compréhension d'une pratique seigneuriale, de son support documentaire et de son potentiel pour la recherche.

Mise à part la présentation de la pratique des papiers-terriers, notre analyse se fonde sur un seul document, le papier-terrier de la

2 On ne compte plus les études et thèses où les documents seigneuriaux interviennent à divers titres dans l'analyse. Pour une illustration d'une utilisation approfondie d'archives seigneuriales ecclésiastiques, on consultera avec profit: Sylvie Dépatie, Mario Lalancette et Christian Dessureault, Contributions à l'étude du régime seigneurial canadien ([Montréal], Hurtubise HMH [1987]), 292 p. Certaines études ont traité ou utilisé les papiers-terriers seigneuriaux de façon spécifique: Fernand Ouellet, «Répartition de la propriété foncière et types d'exploitation agricole dans la seigneurie de Laprairie durant les années 1830", Éléments d' histoire sociale du Bas-Canada (Montréal, Hurtubise HMH, 1972), 113-149; Richard Chabot, «Les terriers de Nicolet: une source importante pour l'histoire rurale du Québec au début du XIX' siècle», Cahiers nicolétains, 6,3 (septembre 1984): 114-126.

3 Albert Soboul, «De la pratique des terriers à la veille de la Révolution», Annales ESC, 19,6 (nov.-déc. 1964): 1049-1051.

4 Chabot, «Les terriers de Nicolet...». 
seigneurie de la Bouteillerie ou de la Rivière-Ouelle datant de $1771^{5}$. Bien qu'une telle approche soit la seule possible dans le cadre actuel de nos connaissances sur ce type de document, elle pose implicitement le problème de la représentativité du document choisi et de la portée des conclusions que nous pouvons en tirer. Afin d'éviter tout malentendu, nous prendrons soin de bien distinguer les éléments spécifiques du document retenu pour l'analyse de ceux qui semblent communs à l'ensemble des papiers-terriers seigneuriaux. Nous croyons cependant que la démarche monographique se justifie de deux autres manières. En premier lieu, la nature même des papiers-terriers seigneuriaux implique une certaine homogénéité de leur contenu, quels que soient le lieu ou l'époque de leur confection. Deuxièmement, nous estimons que l'analyse d'un papier-terrier seigneurial peut largement bénéficier d'une bonne connaissance du terroir en question, ce qui implique nécessairement une approche monographique ${ }^{6}$.

\section{1 - UNE PRATIQUE DE GESTION SEIGNEURIALE}

L'administration d'une seigneurie implique nécessairement le recours à des documents de gestion plus ou moins élaborés. Le papierterrier représente l'un de ces instruments, sans doute le plus fondamental. Un papier-terrier seigneurial est un document qui rassemble les déclarations et reconnaissances des censitaires relatives à leurs possessions foncières dans un fief et aux charges et redevances envers le seigneur. D'un point de vue administratif, un tel document possède une valeur indéniable: il permet au seigneur de faire le point de façon précise sur l'identité de ses débiteurs et sur les montants auxquels il a droit. Par contre, le papier-terrier devient rapidement un document dépassé, dans la mesure où les mutations de la propriété foncière à l'intérieur de la seigneurie modifient sans cesse les informations qu'il contient. C'est pour cette raison, entre autres, que les seigneurs sont autorisés à procéder à la réfection de leur papier-terrier tous les trente ans et ce, aux frais des censitaires ${ }^{7}$.

5 «1771-Papier terrier de la seigneurie de la Bouteillerie, paroisse de la RivièreOuelle», ANQQ, Collection de pièces judiciaires et notariales, $69^{\circ}$ liasse, $\mathrm{n}^{\circ} 17$. Il s'agit d'un manuscrit de 37,5 centimètres sur 25 dont les quelque 150 feuillets sont retenus par une ficelle.

6 Dans notre thèse de doctorat, nous avons eu l'occasion d'étudier le peuplement de la seigneurie de la Rivière-Ouelle: Alain Laberge, «Mobilité, établissement et enracinement en milieu rural: le peuplement des seigneuries de la Grande Anse sous le régime français, 1672 . 1752», thèse de doctorat (histoire), York University, 1987, $323 \mathrm{p}$.

7 Guyot, Répertoire universel et raisonné de jurisprudence civile, criminelle, canonique et bénéficiale (Paris, Panckoucke et Visse, 1783), tome 61: 177. 
Au Canada, la pratique du papier-terrier s'implante graduellement à mesure que progresse le peuplement des seigneuries. En effet, tant qu'une seigneurie reste peu développée, il n'y a aucune nécessité de confectionner un tel document. Il arrive cependant un moment où la confusion des titres est telle qu'un papier-terrier devient indispensable à la gestion seigneuriale. Pour les seigneuries les plus anciennement peuplées, on peut trouver des documents de cette nature dès le dernier quart du XVII ${ }^{e}$ siècle ${ }^{8}$. Mais pour la majorité des fiefs de la vallée du Saint-Laurent, il semble bien qu'il faille attendre le second quart du XVIII' ${ }^{e}$ siècle pour voir se répandre cette pratique.

L'implantation de la pratique des papiers-terriers seigneuriaux a très probablement été influencée par la confection du papier-terrier général de la colonie, une opération qui s'amorce à partir de $1723^{\circ}$. On sait qu'à cette occasion, les seigneurs canadiens ont dû prêter foi et hommage au roi et fournir les aveux et dénombrements de leurs fiefs qui comprennent les renseignements suivants: les noms des censitaires, les dimensions de leurs terres, les bâtiments qui s'y trouvent, le nombre d'arpents labourables et en prairies et le montant des cens et rentes. La façon dont les seigneurs s'y sont pris pour compiler ces données a pu varier. Plusieurs, dont certainement les détenteurs de fiefs très petits ou peu peuplés, n'ont sans doute pas eu à mettre en œuvre des moyens très élaborés pour dresser leurs aveux et dénombrements ${ }^{10}$. D'autres seigneurs ont peut-être tiré ces informations des déclarations des censitaires obtenues à cette fin. Enfin, certains seigneurs ont choisi de procéder à la confection d'un papier-terrier particulier de leur fief afin de pouvoir produire les aveux et dénombrements nécessaires au papier-terrier général de la colonie ${ }^{11}$. Même si cette dernière façon de procéder reste exceptionnelle, la confection du papier-terrier de la colonie était certainement de nature à permettre à plusieurs seigneurs de prendre conscience de la nécessité de mettre de l'ordre dans l'administration de leurs fiefs.

8 Noël Baillargeon, Le Séminaire de Québec sous l'épiscopat de $M^{{ }^{r}}$ de Laval (Québec, Presses de l'Université Laval, 1972), 199.

9 Jacques Mathieu, Alain Laberge, Renald Lessard et Lina Gouger, «Les aveux et dénombrements du Régime français (1723-1745)", Revue d'histoire de l'Amérique française, 42,4 (printemps 1989): 545-562.

10 Dans son étude de la seigneurie de la Rivière-du-Sud, Thomas Wien avance que le seigneur a probablement dressé l'aveu et dénombrement de son fief à l'aide d'informations déjà disponibles, complétées par des observations ponctuelles, plutôt que par le processus de déclarations des censitaires qui risquait de provoquer des résistances. «Peasant Accumulation in a Context of Colonization. Rivière-du-Sud, Canada, 1720-1775», thèse de doctorat (histoire), McGill University, 1988, 102.

11 C'est en tout cas ce qui se passe pour les seigneuries de Montréal et de SaintAugustin. Mathieu, Laberge et al., «Les aveux et dénombrements...», 554 et 556. 
Dans cette perspective de remise à jour de la gestion seigneuriale, le papier-terrier apparaît comme un outil de première importance. Il se distingue d'abord par son mode d'élaboration, qui consiste essentiellement à demander aux censitaires de présenter leurs titres, mais aussi par son caractère judiciaire et légal. En effet, les seigneurs veillent ordinairement à appuyer leur initiative par une requête visant à obtenir une ordonnance des autorités. Cette ordonnance, qui tient lieu de lettres de terrier, se trouve à sanctionner l'opération seigneuriale en lui conférant un caractère contraignant pour le censitaire. De plus, le papier-terrier seigneurial se voit, par le fait même, reconnu comme document légal ayant une pleine valeur devant les cours de justice ${ }^{12}$.

La rédaction du papier-terrier est placée sous la responsabilité d'un notaire qui est commissionné pour ce faire par les autorités ${ }^{13}$. Le rôle du notaire consiste à consigner par écrit les déclarations des censitaires. Malgré l'importance de cette tâche, tant du point de vue du fond que de la forme ${ }^{14}$, l'entreprise de mener à bien la confection du papier-terrier relève plutôt de la personne représentant le seigneur sur les lieux. À la Rivière-Ouelle, par exemple, le négociant Jean-Baptiste Bonenfant agit à ce titre. Il est véritablement le meneur de jeu: c'est lui qui émet l'avertissement général aux censitaires; les déclarations se font chez lui et il détermine l'ordre de comparution des censitaires qu'il va avertir de façon particulière la veille. Mais surtout, Bonenfant a la responsabilité d'accepter ou non les déclarations ${ }^{15}$. Il possède donc une influence déterminante sur la bonne marche des opérations.

12 Un seigneur peut choisir de confectionner un papier-terrier sans recourir à ce type de sanction officielle. R. Chabot signale le cas du terrier de Nicolet de 1806 où le seigneur ne demande pas de lettres de terrier au préalable (Chabot, «Les terriers de Nicolet...», 118). Il est entendu toutefois que, dans ces conditions, la réussite de l'entreprise dépend entièrement de la capacité du seigneur de faire comparaître ses censitaires devant lui pour faire leurs déclarations. Par ailleurs, à défaut d'une valeur légale entière, un tel papier-terrier, s'il est complété, constitue un document de gestion interne aussi valable que celui qui a reçu la sanction officielle. 61: 167 .

13 Le notaire porte alors le titre de «commissaire à terrier». Voir Guyot, op. cit., tome

14 Par sa formation, il est indéniable que le notaire exerce une grande influence sur la manière dont est confectionné le papier-terrier seigneurial. Pour s'en convaincre, on n'a qu'à consulter les documents produits par certains notaires qui semblent s'être "spécialisés» en quelque sorte dans ce genre d'opération. Les papiers-terriers des seigneuries de Saint-Michel (de La Durantaye), Livaudière, Beaupré et Lauzon, confectionnés par le notaire Antoine-J. Saillant entre 1752 et 1765, présentent tous des similitudes évidentes (ANQQ, Greffe de Antoine-J. Saillant, 18 juillet 1752, 8 avril 1756, 1765 (sans date)). Le notaire Saint-Aubin, qui rédige le papier-terrier de la Rivière-Ouelle, a également été responsable de la rédaction de celui de la Pocatière, la seigneurie voisine, quelques mois auparavant. Les deux documents sont en tous points identiques.

15 Guyot, op. cit., 175. 
Un papier-terrier seigneurial se présente donc comme une suite de déclarations de la part de chacun des censitaires de la seigneurie, d'après l'ordre des terres à partir du premier rang ${ }^{16}$. Le contenu des déclarations peut varier légèrement d'un document à l'autre, mais on retrouve toujours une information de base relativement uniforme: l'identité du censitaire, la situation, les dimensions et les bornes de la parcelle déclarée, la provenance de la propriété, une référence au titre de concession originale et le montant des cens et rentes. Les principales variations du contenu sont surtout reliées à la provenance de la propriété, qui peut prendre l'allure d'une véritable chaîne de titres remontant jusqu'au premier détenteur de la parcelle, à l'énumération plus ou moins exhaustive des autres charges des censitaires et à la mention de l'état de la terre, soit la superficie en valeur et les bâtiments.

Avec un document aussi riche, le seigneur dispose d'un véritable instrument de gestion. En plus, le papier-terrier seigneurial est de nature à encourager la production de documents connexes tels que des listes alphabétiques de censitaires qui facilitent la perception et l'administration des redevances ${ }^{17}$. À tous les points de vue, l'utilité du papier-terrier seigneurial est incontestable.

Nés de l'initiative seigneuriale, les papiers-terriers ne forment pas une série documentaire distincte, facile à identifier et à consulter. Notre connaissance de l'implantation et de l'évolution de ce document comme pratique de gestion seigneuriale souffre considérablement de la disparition de nombreuses archives seigneuriales, en particulier laïques, et d'un repérage encore incomplet des papiers-terriers éparpillés dans les greffes des notaires. Tant que cet inventaire systématique n'aura pas été fait, il sera difficile d'établir précisément le rythme d'implantation de cette pratique, que ce soit à l'échelle de la vallée

16 Cette façon de procéder, terre par terre, qui détermine l'appellation de «papierterrier» ou "terrier», ne doit pas être considérée comme étant tout à fait stricte. Les déclarations suivent généralement cet ordre, mais s'il arrive qu'un censitaire possède plus d'une terre, cellesci peuvent parfois être déclarées les unes à la suite des autres, quelle que soit leur localisation dans la seigneurie. En fait, plusieurs papiers-terriers seigneuriaux, en tout cas pour ce qui est du XVIII ${ }^{e}$ siècle, semblent constituer un compromis entre les définitions théoriques de "censier» et de «terrier». D'ailleurs, on en trouve plusieurs qui portent un titre équivoque confondant les deux termes. Par exemple, «Censier ou Papier terrié de la seigneurie de Beaupré (1733)», (ASQ, Manuscrit S-165); «Censier général... servant de Papier terrier» (Saint-Michel, 18 juillet 1752; ANQQ, Greffe de Antoine-J. Saillant).

17 Voir par exemple, jointe au papier-terrier de la seigneurie de la Pocatière, une liste contenant les «Noms des tenanciers (..) suivant l'ordre alphabétique et l'état des rentes et servitudes auxquels ils sont assujettis suivant le papier terrier» (ANQQ, Seigneuries, P-240/25). Ce document constitue un véritable censier. 
laurentienne ou de chaque seigneurie, et de tenter de cerner ses modifications dans le temps, le cas échéant.

Ces lacunes ne doivent pas nous faire perdre de vue que l'analyse de ce type de document représente une voie de recherche non négligeable pour l'histoire du monde rural laurentien pré-industriel. Il s'agit là, en effet, de données de première main issues d'une procédure bien établie. De plus, leur fiabilité est en quelque sorte garantie par le contrôle seigneurial inhérent au processus de confection. À partir des données du papier-terrier de la Rivière-Ouelle de 1771 , nous allons examiner d'abord la procédure de présentation des titres et, ensuite, les apports et les limites des papiers-terriers seigneuriaux pour l'étude de la structure agraire et du paysage rural.

\section{2 - LA PRÉSENTATION DES TITRES}

La présentation des titres par les censitaires constitue la pierre angulaire du papier-terrier. L'examen attentif de cette procédure permet, entre autres, d'apprécier la capacité de légitimation de la propriété foncière des censitaires. La présentation des titres fournit également l'occasion de vérifier un certain nombre de pratiques de gestion dans la seigneurie.

La présentation des titres et la confection du papier-terrier seigneurial débutent peu de temps après l'obtention de l'ordonnance obligeant les censitaires à comparaître devant le seigneur. À la Rivière-Ouelle, par exemple, un avertissement général est donné à la porte de l'église paroissiale quatre dimanches d'affilée par le sousbailli de la paroisse. De plus, à chaque jour, le représentant du seigneur se rend avertir les censitaires qui ont à se présenter le lendemain. La confection du papier-terrier ne risque donc pas de passer inaperçue; toute l'opération est annoncée «afin que personne n'en ignore».

Le papier-terrier de la Rivière-Ouelle de 1771 renferme les déclarations de 144 censitaires décrivant un total de 317 parcelles. Le censitaire procède habituellement à sa déclaration «en son nom». Les exceptions à cette règle sont rares: elles concernent évidemment les héritiers mineurs qui sont représentés par leur tuteur, des personnes absentes momentanément ou résidant ailleurs et d'autres qui choisissent de déléguer un parent à leur place. Compte tenu des implications légales de la déclaration, il n'est pas étonnant de constater que les censitaires décident massivement de comparaître en personne.

La présentation des titres se fait en deux étapes. On demande d'abord aux censitaires d'établir la provenance des parcelles. En général, ceux-ci n'ont guère de difficulté à satisfaire à cette exigence, qu'il 
s'agisse d'une donation, d'un achat, d'un échange ou d'une autre forme d'acquisition. Le papier-terrier contient ce renseignement pour plus de $90 \%$ des parcelles. Les choses semblent se compliquer quelque peu quand vient le moment pour le censitaire de donner les références notariales précises. Pour les 286 parcelles dont la provenance est connue $^{18}$, les censitaires sont en mesure de fournir les précisions demandées dans six cas sur dix. En y ajoutant les références incomplètes, on n'atteint toujours que sept parcelles sur $\mathrm{dix}^{19}$. L'absence de références notariales pour la provenance de 83 parcelles apparaît pour le moins problématique quant à la capacité d'un certain nombre de censitaires de légitimer leurs possessions.

Un examen plus attentif montre cependant que ces lacunes correspondent à des modes d'acquisition bien spécifiques reliés à la transmission familiale du patrimoine foncier. En effet, 68 de ces 83 parcelles proviennent d'héritage ou de donation des père et mère des déclarants. Le fait qu'on ne donne pas davantage de détails sur les transactions elles-mêmes semble indiquer qu'une partie du processus de transmission ne passe pas nécessairement devant le notaire ou que (cela n'excluant nullement ce qui précède) ce type d'acquisition possède en quelque sorte sa propre légitimité découlant du caractère familial de la propriété qui ne saurait être remis en question.

Les cas d'héritage et de donation étant, pour cette raison, mis à part, on peut dire que les censitaires de la Rivière-Ouelle se présentent bien préparés pour établir la provenance de leurs terres. On peut sans doute attribuer une partie de ce résultat à la présence constante d'un notaire dans les environs immédiats de la seigneurie, si ce n'est sur son territoire même, depuis le début du XVIII ${ }^{e}$ siècle. S'il est vrai que l'occupation de fait peut parfois constituer un argument pesant, rien ne vaut une bonne transaction notariée pour l'attester.

La seconde étape de la présentation des titres exige des censitaires qu'ils puissent appuyer la propriété de leurs parcelles sur un contrat de concession original ou, à tout le moins, sur un billet quelconque. Cette procédure est particulièrement importante puisqu'elle permet d'établir exactement le montant des cens et rentes dont sont chargées les terres de la seigneurie.

La plupart des censitaires s'acquittent de cette exigence en présentant directement un «titre primitif» ou en y faisant référence de

18 Nous avons écarté de ce calcul les 31 parcelles dont nous ignorons la provenance et pour lesquelles, évidemment, les références notariales n'existent pas.

19 Par référence notariale incomplète, nous entendons celle où le nom du notaire ou la date de la transaction manquent. En général, c'est la date qui pose problème. 
manière explicite, ce qui rejoint $81 \%$ des parcelles. Pour un petit nombre de parcelles, les censitaires, n'ayant pas de titre primitif à exhiber, se rabattent sur les «dires des anciens tenanciers» afin de justifier un certain montant de cens et rentes. Dans l'ensemble donc, les censitaires connaissent bien les redevances qu'ils doivent au seigneur et les documents qui les fixent à l'origine. Cette conclusion rejoint la précédente sur la reconnaissance des modes d'acquisition des parcelles.

Cette clarté relative des titres primitifs tient en bonne partie à la gestion antérieure de la seigneurie. Sans avoir été nécessairement un modèle du genre, il semble que la distribution du territoire seigneurial en censives a été suffisamment supervisée pour éviter une occupation irrégulière de la part des censitaires. Les références aux titres dans le papier-terrier montrent bien qu'à toutes les époques, les concessions ont fait l'objet de billets sous seing privé signés par le seigneur ou ses représentants, billets souvent versés au greffe du notaire local par la suite. Cette situation se trouve ainsi à éliminer les oppositions et contestations causées la plupart du temps par l'absence ou la confusion des titres.

Le représentant du seigneur accorde des délais à ceux qui en demandent pour pouvoir retrouver leurs titres. Il ne refuse aucune déclaration. L'acceptation des titres est toutefois assortie de la demande de passer «titre-nouvel ${ }^{20}{ }$ » dans plus de la moitié des cas. Cette exigence est formulée lorsque le censitaire est incapable de présenter des titres ou si le titre présenté est un ancien billet sous seing privé ou encore quand trop de censitaires font référence à un seul et même titre primitif. Cette dernière situation est assez fréquente à la Rivière-Ouelle où les premières terres concédées étaient de dimensions fort considérables, pouvant aller jusqu'à douze arpents de front. On comprend alors, qu'au bout de plusieurs décennies et après de

20 Le titre-nouvel est un acte notarié constituant une déclaration de reconnaissance seigneuriale de la part d'un censitaire. Il contient ordinairement les mêmes informations qu'un contrat de concession originale, en plus d'une mise à jour des titres de propriété du censitaire, pouvant aller jusqu'à une chaîne de titres complète. À défaut d'avoir retrouvé le terrier de Mount Murray de 1822 et celui de Murray Bay de 1823-1824, Mario Lalancette utilise les titres-nouvels qui auraient servi à leur rédaction dans son étude de la propriété foncière à La Malbaie, «Essai sur la répartition de la propriété foncière à La Malbaie, au pays de Charlevoix», François Lebrun et Normand Séguin, dir., Sociétés villageoises et rapports villes-campagnes au Québec et dans la France de l'Ouest XVII'-XX' siècles. Actes du colloque franco-québécois de Québec (1985) (Trois-Rivières/Rennes, Centre de recherche en études québécoises, Université du Québec à Trois-Rivières/Presses universitaires de Rennes 2, 1987), 68. De son côté, André Larose associe pratiquement le papier-terrier à un rassemblement de titres-nouvels, «La seigneurie de Beauharnois, 1729-1867: les seigneurs, l'espace et l'argent», thèse de doctorat (histoire), Université d'Ottawa, 1987, chapitre 6. 
multiples mutations, de nombreux censitaires se retrouvent sur la même concession originale. Le titre-nouvel sert donc à effectuer une mise à jour des titres sur une base individuelle.

La présentation des titres permet enfin de s'interroger sur un aspect déterminant de la gestion seigneuriale, soit la perception des diverses charges supportées par les censives, en particulier les redevances foncières comme les cens et rentes, et les lods et ventes à payer à la suite d'une mutation foncière. À ce chapitre cependant, le papierterrier de la Rivière-Ouelle apparaît plutôt décevant.

Le document fait évidemment référence aux cens et rentes dans chacune des déclarations des censitaires. La plupart du temps cependant, le rédacteur se contente de reprendre les termes du titre primitif en ce qui a trait aux montants à payer par arpent de front. Il est rare que le compte précis soit effectué. Les éléments d'information relatifs aux cens et rentes ne semblent donc pas avoir été traités de manière systématique par les responsables de la confection du papier-terrier en vue de clarifier et de faciliter la perception future de ce type de redevances. Sans écarter la possibilité que ces calculs aient été faits et reportés sur une liste de censitaires, le papier-terrier de la RivièreOuelle, tel qu'il se présente, se prête mal à une évaluation de ce chapitre des revenus seigneuriaux.

Il n'est pas du tout question des lods et ventes dans le papierterrier de la Rivière-Ouelle. D'ailleurs, les déclarations ne vont pas audelà de la dernière mutation. En effet, on ne demande pas aux censitaires de refaire complètement la chaîne de titres de leurs possessions foncières, ce qui aurait pu permettre au représentant du seigneur de vérifier si les lods et ventes avaient bien été acquittés. Cette particularité du document, qui ne lui est d'ailleurs pas exclusive, peut s'interpréter de deux manières. En premier lieu, il se peut que la perception de ce droit se soit effectuée normalement, probabilité cautionnée par la présence constante d'un représentant seigneurial sur les lieux, et qu'il n'ait donc servi à rien de consigner toutes les mutations passées. Dans le cas contraire, on doit penser que le seigneur a choisi de renoncer à percevoir les lods et ventes en raison de la prescription pour les mutations intervenues il y a plus de trente ans et du risque de susciter des déclarations évasives de la part des propriétaires de parcelles qui ne seraient pas en règle. Quoi qu'il en soit, il est certain que la confection du papier-terrier de la Rivière-Ouelle ne vise pas particulièrement le recouvrement des lods et ventes et, de ce fait, c'est toute une tranche des revenus seigneuriaux qui nous échappe.

Comme le papier-terrier de la Rivière-Ouelle ne précise pas non plus les autres charges des censitaires, il s'agit d'un document passa- 
blement limité pour l'étude des droits seigneuriaux et l'évaluation du revenu potentiel de la seigneurie. L'utilité du papier-terrier dans ce secteur de recherche est donc directement reliée au degré de précision du document en ce qui a trait à ce type de données.

$\mathrm{Au}$ total, la procédure de présentation des titres permet de scruter plusieurs aspects relatifs à l'opération formelle de confection d'un papier-terrier seigneurial. Il est intéressant de constater que cette analyse rejoint passablement bien le censitaire, appréhendé comme propriétaire devant légitimer ses possessions foncières. Cependant, certains aspects de la gestion seigneuriale, qui tendent à rester dans l'ombre en raison du contenu spécifique du document, sont plus difficiles à saisir.

\section{3 - LE PAPIER-TERRIER ET LA STRUCTURE AGRAIRE}

Bien qu'il soit avant tout un instrument de la gestion seigneuriale, le papier-terrier contient, de par son objet même, des informations sur les principales composantes de la structure agraire. Comme le papierterrier s'intéresse exclusivement aux propriétaires des terres, la propriété foncière constitue un volet privilégié de l'analyse. Le papierterrier de la Rivière-Ouelle de 1771 contient en plus des renseignements qui permettent d'appréhender la capacité de production des censitaires de la seigneurie à cette époque. Ce deuxième volet de l'analyse n'est toutefois pas possible à partir de tous les papiersterriers, plusieurs n'offrant pas ce type de données. Il faut souligner tout de même que le papier-terrier de la Rivière-Ouelle n'est pas unique en son genre: un grand nombre d'autres papiers-terriers seigneuriaux, en particulier au $\mathrm{XVIII}^{\mathrm{e}}$ siècle, indiquent eux aussi la superficie exploitée sur chaque parcelle ${ }^{21}$.

\section{A - La propriété foncière ${ }^{22}$}

Le papier-terrier de la Rivière-Ouelle facilite considérablement l'étude de la propriété foncière en identifiant très précisément les propriétaires de terres. Les cas d'homonymie sont vite réglés par les mentions de l'âge des individus et, souvent, par celles des liens de parenté existant entre eux. Plusieurs papiers-terriers seigneuriaux ne sont pas aussi précis, particulièrement en ce qui concerne l'âge des

21 Peut-être faut-il voir là l'influence de la confection du papier-terrier général de la colonie des années 1720 ou encore celle d'un terroir toujours en expansion?

22 Par sa nature, le papier-terrier ne s'intéresse évidemment pas aux domaines que le seigneur pourrait posséder sur sa seigneurie. Par propriété foncière, nous entendons donc celle relative à la mouvance. 
censitaires. Comme nous le verrons plus loin, la présence de cette dernière variable permet de raffiner l'analyse de la propriété foncière.

Les propriétaires des terres en censive résident la plupart du temps à la Rivière-Ouelle ${ }^{23}$. Presque tous les propriétaires que le document identifie comme étant de l'extérieur (14 cas) demeurent à proximité, surtout dans les paroisses voisines de Sainte-Anne-de-la-Pocatière et de Saint-Roch-des-Aulnaies. On retrouve également un propriétaire résidant à Trois-Pistoles, un autre - le curé Dosquet - à Québec et enfin un dernier domicilié dans le district de Montréal. Somme toute, la propriété foncière reste, à quelques exceptions près, entre les mains des habitants de la Rivière-Ouelle. Propriété foncière presque exclusivement paysanne également. On trouve peu d'autres désignations que celle d' «habitant»: deux négociants, un arpenteur, un maîtretaillandier, un navigateur.

La répartition du nombre de parcelles selon les propriétaires (tableau 1) laisse voir que la moyenne de 2,2 terres par déclarant ne donne pas une image réaliste de la situation. En fait, la moitié des censitaires $(73 / 144 ; 50,7 \%)$ ne possèdent qu'une seule parcelle et les trois quarts $(106 / 144 ; 73,6 \%)$ en ont deux ou moins. La propriété de cinq parcelles et plus n'est le fait que de 14 individus et seulement

\section{TABLEAU 1}

Répartition des censitaires selon le nombre de parcelles et la superficie totale de leur propriété

\begin{tabular}{ccrrrrrrr}
\hline $\begin{array}{c}\text { Nombre de } \\
\text { Parcelles }\end{array}$ & $\mathbf{- 1 0}$ & $\mathbf{1 0 - 4 9}$ & $\mathbf{5 0 - 9 9}$ & $\mathbf{1 0 0 - 1 9 9}$ & $\mathbf{2 0 0 - 2 9 9}$ & $\mathbf{3 0 0 - 4 9 9}$ & $\mathbf{5 0 0 +}$ & Total \\
\hline 1 & 6 & 15 & 27 & 20 & 5 & 0 & 0 & 73 \\
2 & 0 & 2 & 15 & 10 & 5 & 1 & 0 & 33 \\
3 & 0 & 0 & 5 & 8 & 1 & 0 & 0 & 14 \\
4 & 0 & 0 & 4 & 1 & 1 & 4 & 0 & 10 \\
5 & 0 & 1 & 1 & 1 & 1 & 1 & 0 & 5 \\
6 & 0 & 0 & 0 & 1 & 2 & 1 & 1 & 5 \\
7 & 0 & 0 & 0 & 1 & 0 & 0 & 0 & 1 \\
8 & 0 & 0 & 0 & 0 & 1 & 0 & 0 & 1 \\
10 & 0 & 0 & 0 & 0 & 0 & 1 & 0 & 1 \\
16 & 0 & 0 & 0 & 0 & 0 & 0 & 1 & 1 \\
Total & 6 & 18 & 52 & 42 & 16 & 8 & 2 & 144 \\
\hline
\end{tabular}

23 L'information est fournie pour 79 des déclarants (54,9\%). Dans une trentaine de cas, nous ne disposons pas de ce renseignement, mais on peut déduire d'après les patronymes, le type de terre et surtout la présence d'une maison qu'il s'agit effectivement de résidents de la seigneurie. 
deux d'entre eux en accaparent dix et plus. Il est intéressant de noter que celui qui possède le plus grand nombre de terres de la seigneurie n'est nul autre que Jean-Baptiste Bonenfant, négociant et représentant seigneurial, propriétaire de 16 parcelles.

Mais, à lui seul, le nombre de parcelles possédées dit somme toute peu de choses sur le niveau de concentration de la propriété foncière. Une parcelle peut être très grande ou très petite. C'est pourquoi il importe de considérer la superficie des terres. À ce chapitre cependant, le papier-terrier de la Rivière-Ouelle se trouve quelque peu limité. Le problème vient surtout de 54 parcelles dont la profondeur est donnée de manière très imprécise dans le document par des mentions du genre: «sur la profondeur qu'il peut y avoir...» et qui empêchent donc le calcul exact de la superficie. Afin de remédier à cette situation, nous avons alloué une profondeur approximative aux terres concernées en tenant compte de celle des parcelles voisines. Ces corrections vont sans doute causer tantôt des surestimations et tantôt des sousestimations, mais c'était la seule façon de parvenir à mener une analyse d'ensemble d'une facette fondamentale de la propriété foncière.

En juxtaposant le nombre de parcelles et la superficie totale possédés par chacun des censitaires, on peut voir la relation réelle existant entre ces deux éléments. Évidemment, l'accumulation de parcelles favorise celle d'un grand nombre d'arpents de superficie. Les 16 parcelles de Jean-Baptiste Bonenfant totalisent exactement 570,60 arpents de superficie et en font le second plus gros propriétaire de la Rivière-Ouelle. Encore n'est-il pas nécessaire de réunir autant de parcelles: les 863,80 arpents de Joseph Boucher sont répartis entre six terres seulement. Des 26 censitaires avec plus de 200 arpents en superficie, on remarque que seulement cinq d'entre eux n'ont qu'une parcelle et que ceux-ci se situent tous dans le groupe possédant de 200 à 299 arpents (tableau 1).

À l'inverse cependant, la possession de plusieurs parcelles n'implique pas toujours une propriété très étendue. Pour plusieurs censitaires, l'acquisition de parcelles supplémentaires permet en premier lieu d'accéder à un seuil acceptable de superficie. Cette affirmation se vérifie de façon significative dans le cas des propriétaires de 50 à 100 arpents de superficie. Près de la moitié d'entre eux $(25 / 52 ; 48,1 \%)$ ont besoin de plus d'une parcelle pour atteindre ce palier, certains devant aller jusqu'à trois, quatre et même cinq lopins de terre pour y parvenir.

Selon les données du papier-terrier et nos estimations, il y aurait entre 18 et 19000 arpents de superficie concédés en censive à la Rivière-Ouelle en 1771, pour une moyenne de près de 130 arpents par propriétaire. La répartition de ces derniers selon la superficie totale 
possédée (tableau 1) montre cependant l'existence d'un éventail très large de conditions. Le groupe le plus nombreux est celui des 52 propriétaires de 50 à 100 arpents en superficie qui représentent $36,1 \%$ des censitaires. Ce premier groupe est suivi de près par celui des 100 à 199 arpents, soit 42 individus ou 29,2\%. C'est dire que pour rejoindre une majorité de propriétaires, on doit aller jusqu'à 150 arpents de superficie. De plus, on remarque un nombre comparable de censitaires possédant moins de 50 arpents et plus de 200. Dans ces conditions, on ne peut guère parler d'un modèle standardisé de la propriété foncière paysanne. Après un siècle d'occupation du sol, la seigneurie de la Rivière-Ouelle présente un portrait de la propriété foncière qui s'éloigne passablement d'un égalitarisme simpliste entre habitants.

Par l'identification précise des censitaires, le papier-terrier fournit des éléments de distinction entre les diverses conditions de la propriété foncière. Un recoupement de la superficie totale avec le lieu de résidence, par exemple, indique que la très petite propriété de moins de dix arpents demeure presque exclusivement (cinq cas sur six) le fait de résidents de la seigneurie voisine de la Pocatière qui possèdent quelques lopins complémentaires à la Rivière-Ouelle.

Les données patronymiques contenues dans le papier-terrier permettent quant à elles d'évaluer la place relative des divers groupes familiaux dans la structure de la propriété foncière. En 1771, à la Rivière-Ouelle, on compte 36 patronymes isolés et une douzaine d'autres qui reviennent généralement deux ou trois fois chacun. Mais on remarque particulièrement la présence de sept noms de famille qui apparaissent au moins huit fois et qui regroupent à eux seuls 76 censitaires, soit $52,8 \%$ de l'ensemble. Le poids numérique imposant de ces sept familles a son prolongement, avec une proportion plus forte encore, dans la possession du sol: près des trois cinquièmes des parcelles $(186 / 317 ; 58,77 \%)$ et de la superficie concédée $(10912,41$ arpents) leur appartiennent.

L'importance numérique et foncière de ces sept familles découle principalement de l'ancienneté de leur présence dans la seigneurie et de la profondeur de leur enracinement. Ainsi, les Levesque, Bérubé et Hudon sont là dès la décennie 1670 , aux toutes premières heures de la colonisation de la Rivière-Ouelle, tandis que les Dubé, Boucher et Gagnon s'y établissent à la fin du XVII ${ }^{e}$ siècle. L'exemple des Plourde illustre bien la part prise par les alliances matrimoniales dans ce processus: héritiers des Bérubé dès la première génération, deux des fils Plourde de la génération suivante épousent des Levesque. Cependant, l'ancienneté ne joue pas toujours. Le papier-terrier montre également des représentants de familles établies depuis le XVII ${ }^{e}$ siècle, les Dancosse et les Lavoie par exemple, qui sont peu nombreux en 
comparaison avec les sept familles prédominantes et qui se confondent, au plan de la propriété foncière, avec plusieurs autres groupes familiaux installés plus récemment dans la seigneurie.

Lorsque le papier-terrier fournit l'âge des censitaires comme c'est le cas de la Rivière-Ouelle (nous disposons de cette information près de neuf fois sur dix: $126 / 144 ; 87,5 \%$ ), il est possible de pousser plus loin l'étude de la propriété foncière. Cette variable peut être utilisée pour vérifier l'influence du cycle de la vie familiale sur les extensions et les contractions de la propriété foncière paysanne. Le modèle suggère en effet que le patrimoine foncier initial d'une famille va s'accroître en prévision de l'établissement futur des fils et revenir ensuite, au fur et à mesure de leur installation, à la terre principale qui sera elle-même transmise à un des héritiers au moment de la retraite des parents ${ }^{24}$.

Les données du papier-terrier relatives à l'âge en fonction de la propriété foncière montrent que ce modèle se vérifie assez bien dans le cas de la Rivière-Ouelle en 1771. On remarque en effet des variations sensibles de la superficie possédée selon le groupe d'âges (tableau 2). Les 20-29 ans disposent généralement de moins de 100 arpents en superficie ( 20 cas sur $28 ; 71,4 \%$ ) et aucun ne dépasse les 200 arpents. Déjà, dans le groupe des 30-39 ans, les propriétaires de 100 à 200 arpents sont aussi nombreux que ceux de moins de 100 arpents et cinq censitaires possèdent même plus de 200 arpents. Dans la cohorte suivante, celle des 40-49 ans, huit propriétaires contrôlent au moins 200 arpents chacun, dont deux qui en ont plus de 300;

TABLEAU 2

Répartition des censitaires selon le groupe d’âges et la superficie totale de leur propriété*

\begin{tabular}{cccccccc}
\hline & \multicolumn{7}{c}{ Superficie en arpents } \\
Âge & $\mathbf{1 0 - 4 9}$ & $\mathbf{5 0 - 9 9}$ & $\mathbf{1 0 0 - 1 9 9}$ & $\mathbf{2 0 0 - 2 9 9}$ & $\mathbf{3 0 0 - 4 9 9}$ & $\mathbf{5 0 0 +}$ & Total \\
\hline $20-29$ & 1 & 19 & 8 & 0 & 0 & 0 & 28 \\
$30-39$ & 4 & 8 & 12 & 4 & 1 & 0 & 29 \\
$40-49$ & 5 & 8 & 6 & 6 & 2 & 0 & 27 \\
$50-59$ & 1 & 6 & 3 & 1 & 4 & 1 & 16 \\
$60+$ & 2 & 5 & 3 & 1 & 1 & 1 & 13 \\
\hline
\end{tabular}

- Ce tableau rejoint seulement 113 censitaires en raison de l'exclusion des résidents de l'extérieur, des veuves et des mineurs de moins de 20 ans.

24 Gérard Bouchard, «L'étude des structures familiales pré-industrielles: pour un renversement des perspectives", Revue d'histoire moderne et contemporaine, 28 (1981): 568569. 
ensemble ils représentent près de $30 \%$ de ce groupe d'âges. Ce sont les 50-59 ans qui regroupent la plus forte proportion de propriétaires de plus de 200 arpents en superficie $(6 / 16 ; 37,5 \%)$, tous, sauf un, se situant au-dessus de 300 arpents. Enfin, dans la dernière cohorte, celle des plus de 60 ans, les censitaires avec plus de 200 arpents diminuent en nombre et en proportion. En général, ce tableau sommaire semble donc s'accorder avec le modèle proposé.

Le tableau 2 contient cependant des éléments qui appellent des réserves par rapport au modèle. On ne peut s'empêcher de constater la présence d'un nombre non négligeable de censitaires possédant moins de 100 arpents en superficie chez les 30-39 ans et les 40-49 ans. Comme la phase de contraction du patrimoine foncier suite au processus de transmission ne débute habituellement pas à ces âges, cela nous amène à avancer deux explications qui ne s'excluent pas mutuellement. En premier lieu, il est plausible que certains habitants reportent de quelques années les acquisitions foncières nécessaires à l'établissement de leurs enfants pour des raisons pouvant aller d'un nombre restreint de descendants masculins à leurs moyens trop limités. La deuxième explication découle en partie de la première. Elle revient à la notion d'inégalité foncière dont nous avons vu les manifestations un peu plus haut. L'existence même de ces inégalités, qu'on peut remarquer chez tous les groupes d'âge, remet en cause l'application du modèle pour une bonne partie de la société rurale.

Les mentions du mode d'acquisition des parcelles détenues par les censitaires apportent un éclairage supplémentaire en nous renseignant sur les voies d'accès à la propriété foncière (tableau 3 ). On se rend vite compte que deux grandes avenues sont privilégiées: les achats ou échanges et la transmission successorale. Les concessions de terre apparaissent jouer un rôle très mineur, du moins de manière directe. De toute façon, la seigneurie de la Rivière-Ouelle, colonisée depuis un siècle, ne dispose plus d'un grand réservoir de terres disponibles. Dans ces conditions, les concessions de terres nouvelles ne peuvent plus servir de soupape de sécurité en vue de l'établissement des générations futures. Il faut donc se rabattre sur le domaine foncier existant qui impose, comme il se doit, des restrictions importantes à l'accès à

TABLEAU 3

Répartition des parcelles selon le mode d'acquisition

\begin{tabular}{ccccc}
\hline $\begin{array}{c}\text { Transmission } \\
\text { successorale }\end{array}$ & Achat/Échange & Concession & Autres & $\begin{array}{c}\text { Cas } \\
\text { indéterminés }\end{array}$ \\
\hline 109 & 163 & 11 & 3 & 31 \\
\hline
\end{tabular}


la propriété en favorisant les descendants de ceux qui en possèdent déjà des portions appréciables et ceux qui disposent des moyens de les acquérir. Il s'agit là d'un environnement qui nourrit le développement des inégalités foncières.

\section{B - La capacité de production}

L'utilisation des papiers-terriers seigneuriaux pour l'étude de la capacité de production des censitaires ne se fait pas sans problème. Outre le fait que plusieurs de ces documents ne fournissent pas ce type d'information, il y a lieu d'afficher une certaine prudence par rapport à ces données lorsqu'elles s'y trouvent. En effet, comme le papierterrier cherche avant tout à établir la validité des titres de la propriété foncière et des charges qui s'y rattachent, les renseignements relatifs à l'état de développement des diverses parcelles sont purement accessoires. Dans ces conditions, on doit admettre que la qualité générale de ces données risque d'être médiocre et de fournir un tableau plus ou moins exact de la capacité de production.

Dans le cas du papier-terrier de la Rivière-Ouelle, on constate immédiatement l'absence de ces informations pour 49 terres de la seigneurie. Cette situation entraîne nécessairement une sous-estimation de la superficie de terre en labours ou en prairies des 29 propriétaires de ces parcelles. De plus, contrairement aux lacunes touchant la profondeur des terres, cette sous-estimation reste permanente puisqu'il est impossible de la corriger d'aucune manière.

Malgré tout, nous avons quand même cherché à tirer profit des informations disponibles sur la capacité de production des censitaires. Le papier-terrier de la Rivière-Ouelle emploie presque exclusivement la formule «et a dit être sur lad. terre $X$ arpents labourables» pour désigner la superficie cultivable des parcelles. Le total pour l'ensemble de la seigneurie se chiffre à 3502,34 arpents, soit près d'un cinquième de toute la superficie concédée. La répartition des propriétaires selon la superficie labourable (tableau 4) dessine le même large éventail que celui rencontré dans le cas de la superficie totale possédée. La portée de la variation entre deux paliers de superficie labourable devient par contre beaucoup plus significative puisqu'elle affecte directement le niveau de vie en départageant ceux qui sont en mesure de produire des surplus de ceux qui réussissent plus ou moins bien à assurer leur subsistance.

Un regard sur les 21 individus qui possèdent 50 arpents et plus de terre labourable indique bien le profil du paysan aisé de la RivièreOuelle en 1771. À une exception près, ceux-ci disposent toujours de plus d'une parcelle et rassemblent au moins 100 arpents de superficie 
TABLEAU 4

Répartition des censitaires selon la superficie labourable

\begin{tabular}{ccccccccc}
\hline $\begin{array}{c}\text { Nombre d'arpents } \\
\text { labourables }\end{array}$ & 0 & $1-9$ & $10-24$ & $25-49$ & $50-74$ & $75-100$ & $100+$ & Total \\
$\begin{array}{c}\text { Nombre } \\
\text { de censitaires }\end{array}$ & 19 & 27 & 44 & 33 & 12 & 6 & 3 & 144 \\
\hline
\end{tabular}

totale et parfois plus de 500. Cependant, leur âge peut varier considérablement: on en retrouve presque autant dans la trentaine que dans la cinquantaine ou la soixantaine. La principale caractéristique commune (elle vaut pour 16 de ces 21 individus) reste toutefois le fait d'appartenir à l'une des sept vieilles familles mentionnées plus haut qui monopolisaient de façon importante la propriété foncière dans la seigneurie. Ce portrait global confirme donc la présence à la RivièreOuelle d'un groupe de gros exploitants qui se distinguent fortement des autres.

Le papier-terrier est beaucoup moins précis en ce qui a trait aux prairies, la seconde composante de l'exploitation agricole. D'abord, il emploie plusieurs termes pour décrire cette réalité: «prairies», "grèves», «plaines» ou «foins». En second lieu, il ne donne pas toujours le nombre d'arpents qui entrent dans cette catégorie, mais utilise des formules vagues comme «en prairies», «tout en foins», "partie en plaines», etc. Enfin, selon le terrier, seulement 45 censitaires possèdent des arpents en prairies. Ce dernier résultat, qui porte à croire à un phénomène de rareté des prairies à la Rivière-Ouelle, ne peut refléter la réalité ${ }^{25}$. Il y a tout lieu de croire, au contraire, que le papier-terrier néglige de mentionner les arpents en prairies sur les terres qui comptent aussi des arpents en labours, se contentant de relever les parcelles affectées exclusivement à cet usage.

Quoi qu'il en soit, cette présentation sommaire de la capacité de production, même si elle semble aller dans le même sens que nos observations sur la propriété foncière, repose sur des données fragiles et demeure plus superficielle. Par contre, le papier-terrier constitue un indicateur de premier choix quand il s'agit de cerner la répartition de la propriété foncière dans une seigneurie donnée.

25 L'aveu et dénombrement de la scigncuric de la Rivière-Ouelle en 1725 montre en effet qu'une grande majorité des censitaires possèdent des arpents de prairies. (ANQQ, E21/17, ministère des Terres et Forêts, Aveux et dénombrements, Régime français, vol. 1, fol. 233v). 


\section{4 - LE PAPIER-TERRIER ET LE PAYSAGE RURAL}

Par la description et la localisation des terres de la seigneurie, le papier-terrier seigneurial permet de rejoindre divers éléments composant le paysage rural, soit l'environnement tel qu'investi et façonné par les habitants. Bien que la nature et la qualité de l'information varient d'un document à l'autre, le papier-terrier seigneurial constitue souvent la seule source pour tenter de saisir ces réalités sur le terrain même.

Dans le fief de la Rivière-Ouelle, le cœur de la seigneurie, c'est évidemment la rivière Ouelle, sinueuse et dont les larges méandres accentuent encore la présence. Elle regroupe sur ses bords les plus anciennes terres de la seigneurie qui sont également les plus belles, les plus fertiles et les plus développées. C'est autour de la rivière, d'ailleurs, qu'on trouve les fronts les moins larges, souvent limités à moins d'un arpent, et de nombreux petits «circuits» ou terrains comportant tout au plus quelques arpents de superficie. La qualité des terres riveraines en fait des parcelles recherchées, même si elles sont plus petites qu'ailleurs dans la seigneurie. Les terres situées à l'embouchure de la rivière du côté nord-est ont l'avantage supplémentaire de donner également sur le fleuve par la profondeur. Beaucoup plus loin en amont, de nouvelles terres ont été concédées depuis quelques années sur le territoire accordé en augmentation à la seigneuresse en 1750 .

Le deuxième axe d'occupation du sol se situe le long du fleuve Saint-Laurent en allant vers le nord-est. Ce «premier rang», comme il est indiqué dans la marge des déclarations des censitaires du papierterrier de 1771, adopte plutôt une nomenclature variée au gré de la succession des anses et des pointes du littoral fluvial: «Anse aux Iroquois», «Anse à Bouchard», «Pointe aux Orignaux», «Petite Anse», «Pointe Saint-Denis». Les concessions y ont été faites graduellement selon les besoins en terres neuves. En 1771, cette poussée littorale vers le nord-est fait même figure de débordement sur le territoire de la seigneurie voisine de Saint-Denis avec laquelle aucun alignement n'avait été effectué jusqu'alors. Au niveau de la gestion seigneuriale, il semble que l'inertie des seigneurs de Saint-Denis ait profité à ceux de la Rivière-Ouelle qui paraissent retirer effectivement les cens et rentes de ces quelques terres qui ne leur appartiennent pas en réalité ${ }^{26}$.

À partir des années 1730 mais surtout 1740 , des individus se sont faits concéder des terres derrière celles donnant sur le fleuve, dans une

26 Un premier chaînage de la largeur des seigneuries de la Rivière-Ouelle et de SaintDenis a lieu en 1772. Un alignement complet entre les deux fiefs est ensuite effectué en 1774. (ANQQ, Greffe de Ignace Plamondon père, arpenteur, 7 septembre 1772 et 23 septembre 1774). 
zone communément appelée «les Côteaux». C'est une région assez accidentée que domine le "Côteau à chaux» et qui tend elle aussi à rejoindre le fief de Saint-Denis en longeant le «Ruisseau à Bras». Extensions plus récentes du peuplement, comme les dernières terres du premier rang, ces parcelles sont, de plus, désavantagées par une qualité médiocre des sols et par le relief. Toutes ces raisons expliquent leur développement plus inégal.

On distingue une dernière zone d'occupation au sud-ouest de la Rivière-Ouelle dans un triangle coincé entre le fleuve, les terres prenant leur front à l'embouchure de la rivière et le «Grand Ruisseau» qui sert de ligne de démarcation avec la seigneurie de la Pocatière. C'est un lieu où se concentrent de petites parcelles de prairies et de grèves (souvent moins de dix arpents de superficie). Parmi les propriétaires de ces terres, on retrouve la plupart des résidents de la Pocatière qui figurent dans le papier-terrier.

Le papier-terrier de la Rivière-Ouelle fournit également des renseignements sur d'autres aspects du paysage rural de la seigneurie en 1771. Ainsi, à la suite des arpents labourables ou des prairies, les déclarations des censitaires mentionnent ordinairement le nombre et la nature des bâtiments. Si l'on se fie aux données du papier-terrier, la Rivière-Ouelle paraît plutôt sous-équipée. En effet, on ne retrouve jamais plus de deux constructions sur une même parcelle. Il s'agit le plus souvent d'une maison et d'un hangar ou d'une grange. Le terme «étable» n'apparaît qu'une seule fois. Cette situation doit sans doute être mise en relation avec les destructions dont a souffert la Côte-duSud lors de la guerre de la Conquête et dont les habitants se relèvent lentement ${ }^{27}$.

Par ailleurs, le papier-terrier contient des précisions sur le service de bac entretenu pour traverser la Rivière-Ouelle. Il indique de façon indirecte la présence de pêches littorales, d'un «petit moulin», d'une sapinière, et d'une «décharge». Il s'agit là, certes, de détails qui figurent rarement dans les papiers-terriers mais, le cas échéant, ils viennent compléter l'image du paysage rural de cette époque.

\section{CONCLUSION}

Au terme de cet examen du papier-terrier de la Rivière-Ouelle de 1771 , le bilan montre à la fois des forces et des faiblesses dans l'apport de ce type de document à une meilleure connaissance de la

27 Gaston Deschênes, L'année des Anglais. La Côte-du-Sud à l'heure de la Conquête ([Sillery], Septentrion [1988]), 70-72. 
société rurale laurentienne pré-industrielle. Le papier-terrier possède le grand avantage de donner un instantané de la seigneurie à un moment précis de son évolution. Par contre, du fait de son caractère ponctuel, le document ne peut pas cerner les changements à moyen et à long termes.

Le papier-terrier est également un document polyvalent en ce sens qu'il permet de rejoindre plusieurs aspects du monde rural préindustriel. Il est certain, toutefois, que la gestion seigneuriale et la propriété foncière demeurent les deux principaux points de convergence des données d'un papier-terrier. En effet, les papiers-terriers ne fournissent pas tous la capacité de production des parcelles décrites. De toute façon, le cas de la Rivière-Ouelle montre que même lorsque ces renseignements sont présents, ils risquent d'être incomplets et donc de se prêter moins bien à une analyse poussée. Enfin, l'étude du paysage rural repose en grande partie sur la précision qui varie beaucoup elle aussi d'un document à l'autre.

$\mathrm{Au}$ total, le papier-terrier représente un outil d'analyse important que l'on devrait privilégier davantage. Ce faisant, cette pratique seigneuriale sortirait de l'ombre tout en contribuant à une meilleure perception du rapport à la terre dans la société rurale pré-industrielle. 